\title{
Blocking barrier and leaning hill
}

\author{
Chang-Ju Kim (Did htps://orcid.org/0000-0003-4749-5795
}

We are surrounded by many barriers. From individual space to the border, the barriers hold us. In some ways, these barriers are necessary for protecting individuals and for group identity. However, the higher the barrier, movement and thought are more limited. The barriers that penetrate into the realms of other beings have repeated many disputes and tragedies. The barriers of life and thought have caused religious and ideological conflicts.

How about lowering the barrier to become a hill? Lowering the barrier creates opportunity for conversation with neighbors. Lowering barrier increases your chance of helping each other.

Exercise rehabilitation can be set as a new discipline by lowering the barrier of many disciplines such as physical education, health science, medicine, biology, history, and education. Journal of Exercise Rehabilitation aims to create a space where these diverse disciplines can communicate.

In this issue of the Journal of Exercise Rehabilitation, a number of articles are published. There are three articles on the patients with human immunodeficiency virus infection. This seems to be very meaningful to the social role of the exercise rehabilitation. Many valuable results of exercise rehabilitation on the physical disabili- ties have been published. The effects of exercise rehabilitation on the mental and cognitive abilities have also been included.

We can see that the Journal of Exercise Rehabilitation has fulfilled its role of integrating and converging many disciplines. We expect that the convergence of many different disciplines will give new opportunities to overcome many intractable diseases. The Journal of Exercise Rehabilitation will continue to evolve into a hill where many disciplines can rely on one another.

\section{CONFLICT OF INTEREST}

No potential conflict of interest relevant to this article was reported.

President of the Korean Society of Exercise Rehabilitation Department of Physiology, College of Medicine Kyung Hee University 26 Kyungheedae-ro, Dongdaemun-gu, Seoul 02447, Korea E-mail: changju@khu.ac.kr 